\title{
Stochastic control and real options valuation of thermal storage-enabled demand response from flexible district energy systems
}

\author{
Yerkin Kitapbayev $^{a}$, John Moriarty*, Pierluigi Mancarella ${ }^{b}$ \\ The University of Manchester, Oxford Road, Manchester M13 9PL, UK \\ ${ }^{a}$ E-mail address: yerkin.kitapbayev@manchester.ac.uk \\ * Corresponding author. Tel.: +44 (0) 1612755883. \\ E-mail address: j.moriarty@manchester.ac.uk \\ ${ }^{b}$ E-mail address: p.mancarella@manchester.ac.uk.
}

November 24, 2014

\begin{abstract}
In district energy systems powered by Combined Heat and Power (CHP) plants, thermal storage can significantly increase CHP flexibility to respond to real time market signals and therefore improve the business case of such demand response schemes in a Smart Grid environment. However, main challenges remain as to what is the optimal way to control intertemporal storage operation in the presence of uncertain market prices, and then how to value the investment into storage as flexibility enabler. In this outlook, the aim of this paper is to propose a model for optimal and dynamic control and long term valuation of CHP-thermal storage in the presence of uncertain market prices. The proposed model is formulated as a stochastic control problem and numerically solved through Least Squares Monte Carlo regression analysis, with integrated investment and operational timescale analysis equivalent to real options valuation models encountered in finance. Outputs are represented by clear and interpretable feedback control strategy maps for each hour of the day, thus suitable for real time demand response under uncertainty. Numerical applications to a realistic UK case study
\end{abstract}

with projected market gas and electricity prices exemplify the proposed approach and quantify the robustness of the selected storage solutions.

Keywords. Heat storage, flexibility, demand response, combined heat and power, real options, stochastic control, stochastic process, smart grid

\section{Nomenclature}

\section{Acronyms}

CHP Combined Heat and Power

DR Demand Response

RO Real Options

DCF Discounted Cash Flow

LSM Least Squares Monte Carlo regression

\section{Symbols ${ }^{1}$}

\section{Price models}

\footnotetext{
${ }^{1}$ Principal nomenclature used in the text. Where possible subscripts, superscripts and function notation have been suppressed to aid clarity.
} 


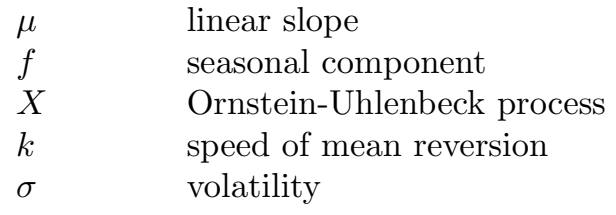

\section{Stochastic control problem}

$\mathbf{u} ; \mathrm{u} \quad$ set of feedback controls; one feedback contr $t ; T \quad$ current time; end time

$r \quad$ financial discount rate

$\psi\left(u_{t}\right) \quad$ net expenditure rate on gas and electricity E mathematical expectation operator $V \quad$ average total operational cost

\section{Dynamic programming}

$\begin{array}{ll}s & \text { time in dynamic program } \\ C & \text { level of stored heat } \\ E ; G & \text { electricity price; gas price } \\ D & \text { system state }(s, G, E, C)\end{array}$

\section{Discretisation and regression}

$c_{1}, \ldots, c_{L}$ heat storage levels $h ; M \quad$ time step size; number of time steps $N \quad$ number of simulated price paths $\xi_{1}, \ldots, \xi_{z}$ operational modes for generation assets $B ; \alpha \quad$ basis functions; regression coefficients

\section{Introduction}

In multi-energy systems [1], different energy vectors are modelled in an integrated manner so as to optimise the overall energy system. Within this context, distributed multi-generation plants [2, 3] and especially Combined Heat and Power (CHP) plants play a key role, particularly to set up district energy systems. While traditionally such plants have been operated and designed in a load-following mode with boiler back-up, new opportunities are arising from active participation in energy markets. More specifically, multi-generation plants that can deploy flexibility from arbitraging between energy vectors can provide real-time Demand Response (DR) $[4,5]$ to external market signals, thus boosting their overall business case with respect to classic "passive" loadfollowing operation. In this outlook, thermal energy storage can provide a significant degree of flexibility to CHP-based systems $[6,7,8,9]$ by allowing decoupling of supply and demand as well as time arbitrage to buy/sell energy to/from markets (gas and electricity) in real-time. However, many uncertainties arise in different time scales for district energy systems, namely, large scale uncertainties mostly from energy price evolution in the planning horizon/long run, and small scale uncertainties from load and energy price variations in the operational horizon/short run (for a comprehensive analysis of these issues, please refer to $[10,11])$. In this context, while engineering techniques often encounter serious challenges to cope with such uncertainty modelling, mathematical finance provides efficient computational techniques that can address uncertainties in both operation and planning aspects concurrently. This is important to capture because short term flexibility can change the long term business case, while the long term investment plan can enable short term flexibility.

On the above premises, following preliminary work done in [12], this paper proposes a model based on stochastic optimal control theory [13] which provides an innovative integrated approach to optimally and dynamically (in the sense of providing real time DR) controlling CHP plants with heat storage in the presence of uncertain market prices, with no requirement for separation of the operational and investment timescales. In fact, the constrained linear stochastic optimal control algorithm used for operational optimisation explicitly considers interactions with measured and predicted external price signals in both the short run and the long run. By doing so, it also performs at the same time Real Options (RO) [14] valuation of thermal storage for planning purposes, thus fully capturing and valuing the flexibility that thermal storage makes available in CHP systems. In this respect, in fact, Discounted Cash Flow (DCF) methods that are traditionally used to value investment projects generally fail to properly model uncertainty and operational flexibility, by adopting the typical solution of increasing the project discount 
rate to account for risk. In contrast, in district energy systems that are capable to provide DR a substantial share of their operational value arises from the fluctuations across energy prices and over different time scales, which are excluded from DCF valuation and for which $\mathrm{RO}$ is most suitable. In this perspective, several applications of RO to energy systems have been provided in the last years, for instance in Australia and New Zealand for network investment projects [15]. Similar examples can be found in $[14,16,17]$. Relevant to the problem discussed here, RO valuation is applied in a multienergy system scheme in [18] by carrying out a series of daily independent optimal scheduling calculations for distributed energy resources; however, this approach does not take into account the effect on scheduling for subsequent time periods (and the relevant opportunity costs), nor optimises DR strategies by taking into account price prediction and feedback from real-time information. In contrast, in the model put forward here, hour-by-hour control solutions are obtained in feedback form [19] (taking into account real-time price information) and do not separate the investment and operational timescales, instead working with a single timescale irrespective of the duration. The price models used are realistic and calibrated to the current market view via inference from quoted UK electricity and gas futures prices. Further, computational efficiency is discussed and achieved through combining forward simulation with regression modelling and backward dynamic programming [20]. A key and innovative output of the proposed model is our use of statistical stationarity to produce high quality real time control strategy maps: in contrast to classical dynamic programming, these are not defined on any discrete lattice but their resolution is adaptive and guided by the statistical price models themselves, deliberately giving higher-quality information in areas of state space which are statistically more likely to occur, while still covering more unlikely system states. These high quality control maps can be automatically built at each considered operational time step and take into account both the long term prediction of market prices as well as real-time price information.

The paper is organised as follows. Section 2 pro- vides an introduction to the relevant control problem and the modelling aspects. Section 3 discusses the Least Squares Monte Carlo Regression (LSM) method used for computationally efficient numerical solution. Section 4 presents a realistic UK based case study application, whose results are shown and discussed in Section 5. Section 6 contains the concluding remarks.

\section{Problem description and modelling}

The value of heat storage lies in its ability to effectively move heat demand through time. In the presence of flexible CHP and energy markets (such as the district energy system in Fig. 1) this creates an opportunity to create financial value, and in particular due to volatile and stochastic market prices. Details of the problem and the model developed are provided below.

\subsection{Price dynamics and the value of heat storage}

The daily fluctuations in prices (gas and electricity) and demand (electricity and heat) used in our case study are plotted in Fig. 2. The demand profiles, given with hourly resolution, were generated through the tool in [21]. Price fluctuations are an indication of the potential value offered through the incorporation of heat storage to increase system flexibility, which may be divided into two main components: gas price arbitrage and exploiting spark spread.

Gas price arbitrage. As gas (and electricity) prices exhibit stochastic variations around their average levels, periods of below-average gas price may be exploited by increasing the heat production rate and storing the produced heat, anticipating that the gas price will revert to (or above) its average level. If the speed of gas price mean reversion is sufficiently high then there is the potential for operating cost reduction by performing gas price arbitrage even in the presence of cumulative heat losses over time. In this case, economic optimisation clearly depends on 


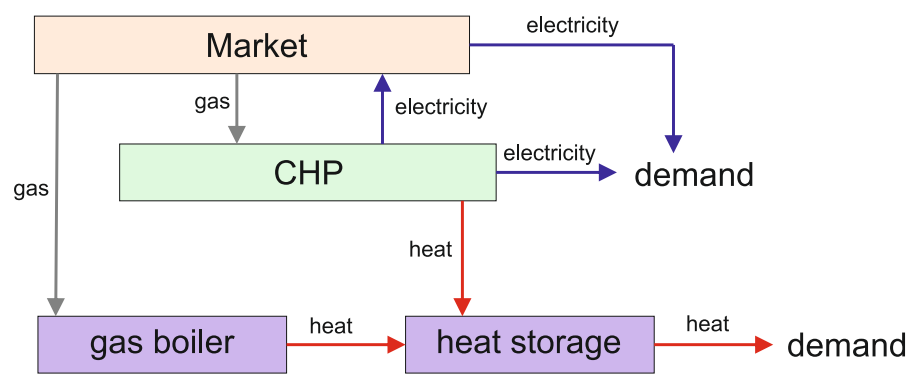

Figure 1: The multi-component district energy system modelled in the case study.

good estimation of the speed at which the gas price reverts towards its mean, for instance using the classical Ornstein-Uhlenbeck model [22]. This is a simple model, having just two parameters in addition to the average price level: the speed $k$ of mean reversion and the volatility $\sigma$ of the stochastic component of the price. The time-varying average price level and these two parameters may be estimated from historic price time series, and this calibration is performed in section 4.3

Exploiting spark spread. It can be seen that the difference between the electricity and gas price (the 'spark spread') has an underlying daily cycle driven by variations in the average electricity price. While a high spark spread may be exploited by operating CHP at its maximum rate, in summer this may result in heat generation in excess of demand. Heat storage, if available and not already full, can therefore shift this 'negative demand' through time to meet later demand, reducing operational cost. In this case, flexibility value is driven not just by price fluctuations around the mean, but also by the average size of the spark spread itself. While this may also be analysed historically, for heat storage investment purposes it is future trends in the spark spread which concern us. If the seasonal component is sufficiently slowly varying (for example, with period one year) then it can be estimated directly from electricity and gas futures prices, and we may infer from these prices the market's view of expected trends over the next few years for which futures prices are quoted. This inference is described and carried out in section 4.4.
Diurnal components, however, must be fitted from historic time series as described above and assumed to persist into the future.

\subsection{Mean-reverting stochastic process models}

Our stochastic model for the hourly movements of electricity and gas prices has three components:

- a deterministic seasonal component $f(t)$,

- a deterministic linear trend $\mu t$, and

- a mean-reverting stochastic component $X(t)$.

Thus if $P(t)$ represents a price at time $t$ (in $£ / \mathrm{MWh}$ ) then we have the (arithmetic) price model

$$
P(t)=f(t)+\mu t+X(t)
$$

The stochastic dynamics of the mean-reverting component $X(t)$ are of Ornstein-Uhlenbeck type:

$$
d(X(t))=-k X(t) d t+\sigma d W(t)
$$

where $k, \sigma$ are parameters representing the speed of mean reversion and the volatility respectively and $W(t)$ is the classical Brownian motion (Wiener) process.

Since price processes are typically non-negative and are often characterised in terms of percentage price movements, a common alternative to the model of Eq. 1 is the following geometric model:

$$
\log P(t)=f(t)+\mu t+X(t)
$$



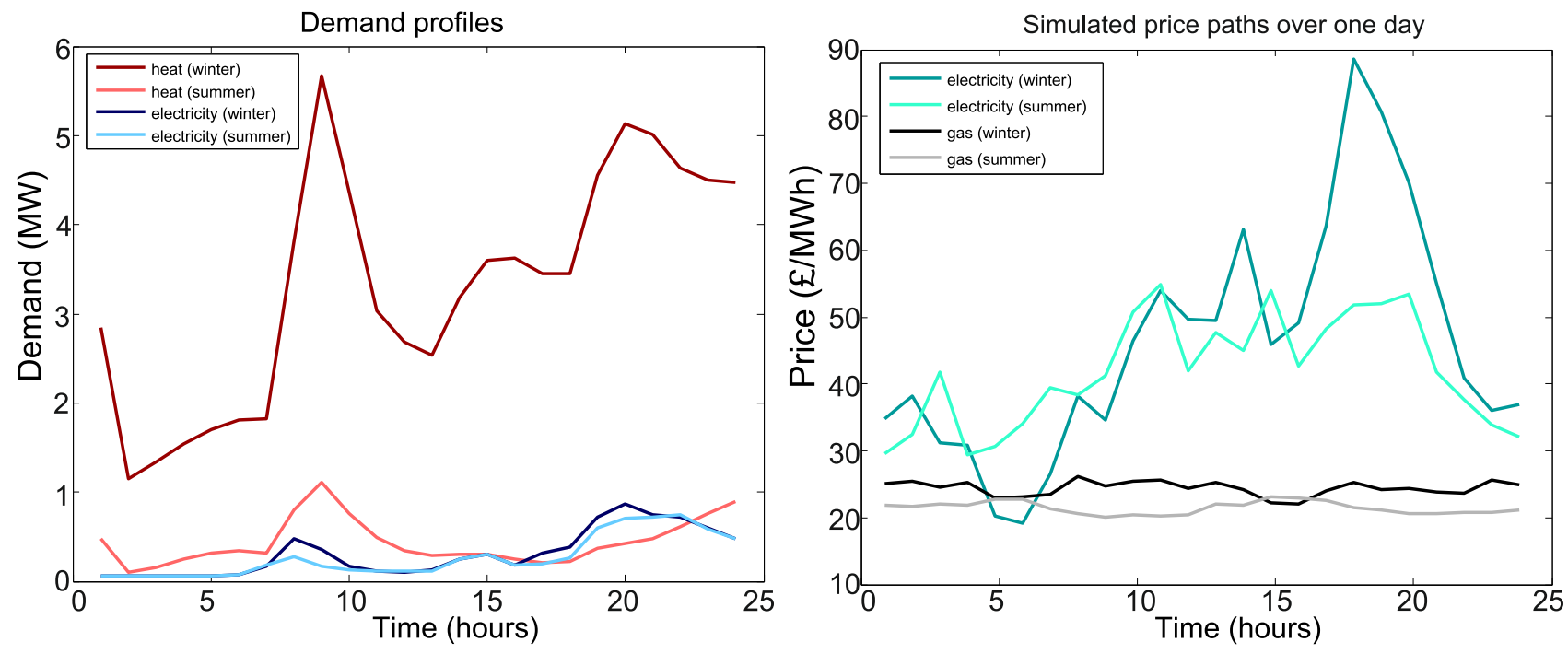

Figure 2: Left: Hourly electricity and heat demand profiles for winter and summer used for the district energy system. These profiles were generated through the tool [21]. Right: Simulated hourly winter and summer price paths over one day. From the electricity spot price model Eq. 3 calibrated as described in section 4.3 we plot simulated paths $\exp (f(t)+\mu t+X(t))$ for 1st January and 1st July 2015. From the gas spot price model Eq. 1 calibrated as described in section 4.3 we plot simulated paths $f(t)+\mu t+X(t)$ for 1st January and 1st July 2015.

During the calibration procedure described below we examine whether the arithmetic model of Eq. 1 or the geometric model of Eq. 3 better explains the quoted futures prices.

\subsection{Stochastic control problem}

The stochastic control problem we address in this paper is the selection of an optimal feedback control policy for the energy system's flexible generation assets to minimise the average long-run operational cost for the district energy system, where operational cost is the total expenditure on electricity and gas, net of any electricity sold back to the grid. A feedback control policy is a map which takes as input the current system state and whose output is an operational state to be applied. The feedback control policy must be admissible in that it must fully meet demand at all times while respecting the physical constraints of the district energy system. We introduce the notation

- $\mathbf{u}$ is the set of all admissible feedback control policies

- $d=(t, g, e, c)$ represents the state of the system at any particular time $t$. The components of $d$ are time, gas price, electricity price and level of stored heat respectively

- $T$ is the time at which our optimisation ends

- $\psi(u(d))$ is the rate of expenditure on both gas and electricity (net of the rate of any electricity sales back to the market) under the particular feedback control policy $u \in \mathbf{u}$ when the system state is $d$

- $V_{u}(d)$ is the expected net present value of the total operational cost for the system from time $t$ to the end time $T$ under the particular control policy $u \in \mathbf{u}$, if the system state at time $t$ is $d$. 
Let $\mathrm{E}^{d, u}$ denote the expectation operator (the superscripts denoting initial system state $d$ and feedback control policy $u$ ). Then from the above definitions we have

$$
V_{u}(d)=\mathrm{E}^{d, u}\left[\int_{t}^{T} e^{-r(s-t)} \psi\left(u_{s}\right) d s\right]
$$

where $u_{s}$ represents the operational control applied at time $s$ (see section 3.1 below for its definition as a stochastic process) and $r$ is the financial discount rate applied in the DCF calculation. Then the average total operational cost under rational management is the minimum

$$
V(d)=\min _{u \in \mathbf{u}} V_{u}(d)
$$

and the rational control policy is the corresponding minimiser

$$
u^{*}(d)=\arg \min _{u \in \mathbf{u}} V_{u}(d)
$$

This defines our optimisation problem.

The solution method we employ, which is an extension of that presented in [20], takes full account of opportunity costs, or the fact that control actions taken at the present moment may introduce additional constraints at later moments, due either to physical impossibilities or realised financial losses. It is also dynamic in the sense that different optimal control maps are produced for each hour in the study. This is achieved efficiently by combining the backwards-intime recursion employed in dynamic programming, which reduces the number of comparisons made during optimisation, with Monte Carlo regression analysis, which allows the stochastic gas and electricity price models (Figs. 2 and 3) to effectively guide the selection of the points at which the operational cost is evaluated, enabling highly precise operational control maps to be constructed within reasonable computational time. The control strategies produced may be used as decision rules in real-time feedback control of the generation assets, where the current storage level and market prices are mapped to the appropriate operational state by the generated optimal control maps (exemplified by Fig. 4). The model is therefore consistent with provision of real-time price signals as well as the use of forecasted energy prices, and is thus suitable to reproduce future real-world operation of information and control technology enabled smart districts. In this real-time operational use case, however, the feedback strategies themselves would be regularly recalculated in order to take account for example of weather prediction-adjusted demand profiles.

\section{Numerical methods: Dy- namic Backward Program- ming and Least Squares Monte Carlo Regression}

\subsection{Notation for stochastic processes}

In order to describe the optimisation procedure notation is required for the (stochastic) state of the system at each later time $s>t$ :

- Let $E_{s}^{d}, G_{s}^{d}$ denote the stochastic electricity price and gas price respectively at time $s$, given that the initial state was $d$

- Let $C_{s}^{d, u}$, denote the level of stored heat at time $s$, given that the initial state was $d$, under the control policy $u$

- Let $D_{s}^{d, u}=\left(s, G_{s}^{d}, E_{s}^{d}, C_{s}^{d, u}\right)$ and $u_{s}=u\left(D_{s}^{d, u}\right)$ be the state of the system and the operational mode respectively at time $s$, given that the initial state was $d$, under the control policy $u$.

\subsection{Discretisation}

We interpret Eq. 4 in discrete time and with discrete states. We also effectively discretise the probability measure governing the stochastic processes in section 3.1 by independently drawing a fixed number of sample paths. That is:

- fix a time step $h$ and number of steps $M=T / h$

- discretise the heat storage level into $L$ equally spaced possible levels $c_{1}, \ldots, c_{L}$ 
- simulate price pairs $\left(g_{m h}^{n}, e_{m h}^{n}\right)$ for $n=1, \ldots, N$, $m=1, \ldots, M$ and define the system states $d_{m}^{n, j}=$ $\left(m h, g_{m h}^{n}, e_{m h}^{n}, c_{j}\right)$ for $j=1, \ldots, L$

- suppose that we have possible operational modes $\xi_{1}, \ldots, \xi_{z}$ for our generation assets. Let $u_{i}$ denote the trivial feedback control policy which simply maintains operational mode $\xi_{i}$ at all times, irrespective of the system state.

Using this discretisation we now describe our backwards induction procedure.

\subsection{Backwards induction}

Since with no time remaining there is no operational cost we have $V_{u_{i}}(M, g, e, c)=0$ for any prices $g, e$, storage level $c$, and operational mode corresponding to $u_{i}$. We will compute by backward induction the value functions and optimal operating modes

$$
V\left(d_{m}^{n, j}\right):=\min _{i} \mathrm{E}_{m}^{d_{m}^{n, j}, u_{i}}\left[h \psi\left(\xi_{i}\right)+e^{-r h} V\left(d_{m+1}^{n, \ell}\right)\right] \mathbf{4} \text { Case study application }
$$

\section{Case study application}

$u^{*}\left(d_{m}^{n, j}\right):=\arg \min _{i} \mathrm{E}^{d_{m}^{n, j}, u_{i}}\left[h \psi\left(\xi_{i}\right)+e^{-r h} V\left(d_{m+1}^{n, \ell}\right.\right.$ In this case study we consider the flexible district

for $m=M-1, \ldots, 0, n=1, \ldots, N, j=1, \ldots, L$, where $c_{\ell}$ is the new level of heat storage when operating mode $\xi_{i}$ is applied in the system state $d_{m}^{n, j}$. The central question is to compute the conditional expectations

$$
\mathrm{E}^{d_{m}^{n, j}, u_{i}}\left[V\left(d_{m+1}^{n, \ell}\right)\right]
$$

Using the approach of [13] we approximate (8) by a linear combination of basis functions $B_{k}$ :

$$
\mathrm{E}^{d_{m}^{n, j}, u_{i}}\left[V\left(d_{m+1}^{n, \ell}\right)\right] \approx \sum_{k=1}^{N_{b}} \alpha_{k}(i, j, m) B_{k}\left(g_{m h}^{n}, e_{m h}^{n}\right)
$$

We choose the basis functions $B_{k}$ as degree 2 polynomial functions so that each such linear combination is given by (writing $\alpha_{k}(i, j, m)=\alpha_{k}$ here for brevity)

$\alpha_{1}+\alpha_{2} g_{m h}^{n}+\alpha_{3} e_{m h}^{n}+\alpha_{4}\left(g_{m h}^{n}\right)^{2}+\alpha_{5} g_{m h}^{n} e_{m h}^{n}+\alpha_{6}\left(e_{m h}^{n}\right.$ and may be fitted by least squares regression, so that the vector $\alpha=\left(\alpha_{k}\right)_{k=1, \ldots, 6}$ is given by

$$
\alpha=\left(Q^{T} Q\right)^{-1} Q y
$$

where

$$
Q=\left(\begin{array}{cccccc}
1 & g_{m h}^{1} & e_{m h}^{1} & \left(g_{m h}^{1}\right)^{2} & g_{m h}^{1} e_{m h}^{1} & \left(e_{m h}^{1}\right)^{2} \\
\vdots & \vdots & \vdots & \vdots & \vdots & \vdots \\
1 & g_{m h}^{N} & e_{m h}^{N} & \left(g_{m h}^{N}\right)^{2} & g_{m h}^{N} e_{m h}^{N} & \left(e_{m h}^{N}\right)^{2}
\end{array}\right)
$$

and the vector $y=\left(y_{n}\right)_{n=1, \ldots, N}$ is given by

$$
y_{n}=V\left(d_{m+1}^{n, \ell}\right)
$$

Hence using (11) we approximate the conditional expectations (8) and the backward-in-time recursion may be completed for $m=M-1, \ldots, 0$. shown in Fig. 1 and described in section 4.1. Section 4.2 describes the UK market data used, while details of the calibration of these models to historic and futures prices are provided in sections 4.3 and 4.4 .

\subsection{Modelling of district energy sys- tem}

The specific model of a flexible district energy system that we consider here is illustrated in Fig. 1. The components of the system are a CHP unit, gas boiler, heat storage and heat network. Local heat demand, which is aggregated through a heat network, must be satisfied at each hour of the day by a combination of storage, boiler and CHP heat output and local hourly electricity demand must also be satisfied, by the market and CHP electricity output. Gas and electricity markets are accessible through a network connection and electricity may be sold back to the market. We ${ }_{h} y^{2}$ se the following notation and specific numerical assumptions for the case study under consideration: 
Table 1

Operating modes for the multi-energy system shown in Fig. 1.

\begin{tabular}{llll}
\hline \hline & CHP & & Boiler \\
Regime & Input (Gas, MW) & Regime & Input (Gas, MW) \\
C1 & 0 & F1 & 0 \\
C2 & 1.875 & F2 & 1.65 \\
C3 & 3.75 & F3 & 3.3 \\
C4 & F4 & 4.95 \\
C5 & 5.625 & F5 & 6.6 \\
\hline \hline
\end{tabular}

- The amount of heat in storage at time $t$ is $C_{t}$ (in MWh), with the physical constraint $C_{t} \leq$ $C_{\max }$. Our central case study takes $C_{\max }$ as 15 and in sensitivity analysis we also consider values for $C_{\max }$ of $22.5,7.5$ and 0 . The case $C_{\max }=0$ provides a baseline with which we may assess the value of the storage unit in the context of this flexible district energy system. Heat in storage undergoes standing losses of $0.5 \%$ per hour.

- The conversion efficiency of the CHP unit (from gas) is $e_{1}=0.4$ to electricity and $h_{1}=0.4$ to heat, and the efficiency of the gas boiler is $h_{2}=$ 0.91. Efficiencies are all assumed constant for the sake of simplicity, but output-related models could be readily incorporated in the proposed approach.

- The maximum gas input rates are 7.5 MW for CHP and 6.6 MW for the boiler (so as to be able to cover the whole heat demand with the boiler in the case of outage of the CHP).

- The gas and electricity markets are spot markets. The buy-price for gas is the wholesale price plus a fixed $15 £ /$ MWh network charge, while the buy-price for electricity equals the wholesale price plus a fixed $50 £ / \mathrm{MWh}$ network charge. Electricity may be sold back to the market at the wholesale price.

- The discount rate (for investment value calculations) is $6 \%$ per annum.

- We optimize over the three year period 2015 to 2017 inclusive. This period has been chosen to match the availability of futures prices for calibration of the financial model, and also illustrates the capability of our numerical method to perform optimisation on investment timescales (in addition to its applicability over operational timescales). In order to study longer timescales our analysis may be repeated for a sequence of initial energy prices consistent with the price trends inferred in section 4.4 and the results combined in a standard DCF calculation via discounting.

- The correlation between the driving noises (represented by the term $d W$ in Eq. 2) for electricity and gas prices is 0.4 , which is consistent with the value estimated in [12] from historic prices.

We assume that the system illustrated in Fig. 1 may be operated in a flexible but discrete manner by choosing between the operating regimes given in Table 4.1. A plant control regime C3F5 is made up of a combination of a CHP regime $\mathrm{C} 3$ and a boiler regime F5. For example, regime C3F3 corresponds to both the CHP unit and the boiler operating at half capacity. To model physical ramp rates, it is assumed that regime switches which involve changing the operating state of the CHP unit take effect with a delay of 15 minutes. Finer regimes could be easily implemented at the expense of an increase in computational time, but this limited number of operational states is appropriate in this hour-by-hour optimisation study of a three year operational period.

Since the electricity buy price is always greater than the sell price (as in most normal applications), any electricity output from CHP is always used to 
meet local electricity demand and only surplus electricity is sold into the electricity market. When local electricity demand exceeds the chosen CHP output the deficit is met by buying electricity in the market. Initially no heat is stored, and the change in storage level over any period is equal to the total heat output from the boiler and CHP net of heat demand, minus standing losses.

\subsection{Market data}

The following time series were retrieved from Thomson Reuters Datastream on 26th January 2014:

1. UKPX Reference Price Data from January 2011 to December 2013

2. National Grid Gas System Average Price from January 2011 to December 2013

3. Summer and winter Inter-Continental Exchange UK Base Electricity Futures prices from 2015 to 2018

4. Monthly Inter-Continental Exchange UK Natural gas futures prices from January 2015 to March 2018

The futures prices are plotted in Fig. 3 (for clarity only winter prices are shown in the case of electricity). The trend in electricity futures prices is clearly nonlinear and in fact is well described by the geometric spot price model of Eq. 3, while the arithmetic spot price model of Eq. 1 provides a better fit to the quoted gas futures prices.

\subsection{Calibration of stochastic price components}

Since spot price data illustrates price variation, we use the historic spot price data series indicated above to calibrate the stochastic part $X$ of the price models given in Eqs. 1 and 3, by estimating the parameters $k$ and $\sigma$ in Eq. 2 separately for electricity and gas as follows.

Electricity. We observe (data not shown) that the electricity price data exhibits two clear seasons related by an approximately constant price ratio: winter (October-March) and summer (April-September).
A seasonal component with a period of one day is clearly exhibited and this component ( $f$ in Eq. 3 ) is estimated from the winter UKPX spot price data. The estimated speed of mean reversion in Eq. 2 may then be estimated as $k_{e}=0.25$ and the average squared regression error in Eq. 3 is 0.0198, corresponding to a fitted volatility (standard deviation) in Eq. 2 of $\sigma_{e}=0.14$.

Gas. In contrast to the electricity model, and consistent with the storability of gas, we find that the seasonal component of the gas price has period one year and we estimate this component of the arithmetic spot price model Eq. 1 directly from monthly gas futures prices in section 4.4. From the National Grid Gas spot price series, the fitted value for the speed of mean reversion may then be estimated as $k_{g}=0.25$ and the fitted hourly volatility is $\sigma_{g}=0.95$.

\subsection{Calibration of deterministic price components}

Since we model futures prices as averages, then (provided they are quoted at sufficiently many maturities) they may be used to calibrate the deterministic parts $\mu t$ and $f(t)$ of the price models given in Eqs. 1 and 3 as follows.

Electricity. From the futures price data the summer-winter price ratio was found to be 0.85 . In order to calibrate the linear price trend, the theoretical electricity futures price implied by the geometric model of Eq. 3 is

$$
F_{e}(T)=\exp \left[f(T)+\mu T+X_{0} e^{-k_{e} T}+\frac{\sigma^{2}}{4 k_{e}}\left(1-e^{-2 k T}\right)\right]
$$

From Eq. 14 the annual linear trend was fitted as $\mu_{e}=0.014$ while a value of -0.183 was fitted for the initial value $X_{0}$ of the stochastic component $X$. As can be seen from Fig. 3, the fit between theoretical and observed futures prices is extremely good.

Gas. The theoretical futures prices implied by the arithmetic model Eq. 1 are

$$
F_{g}(T)=f(T)+\mu T
$$

From Eq. 15 we fit a negative linear annual drift 

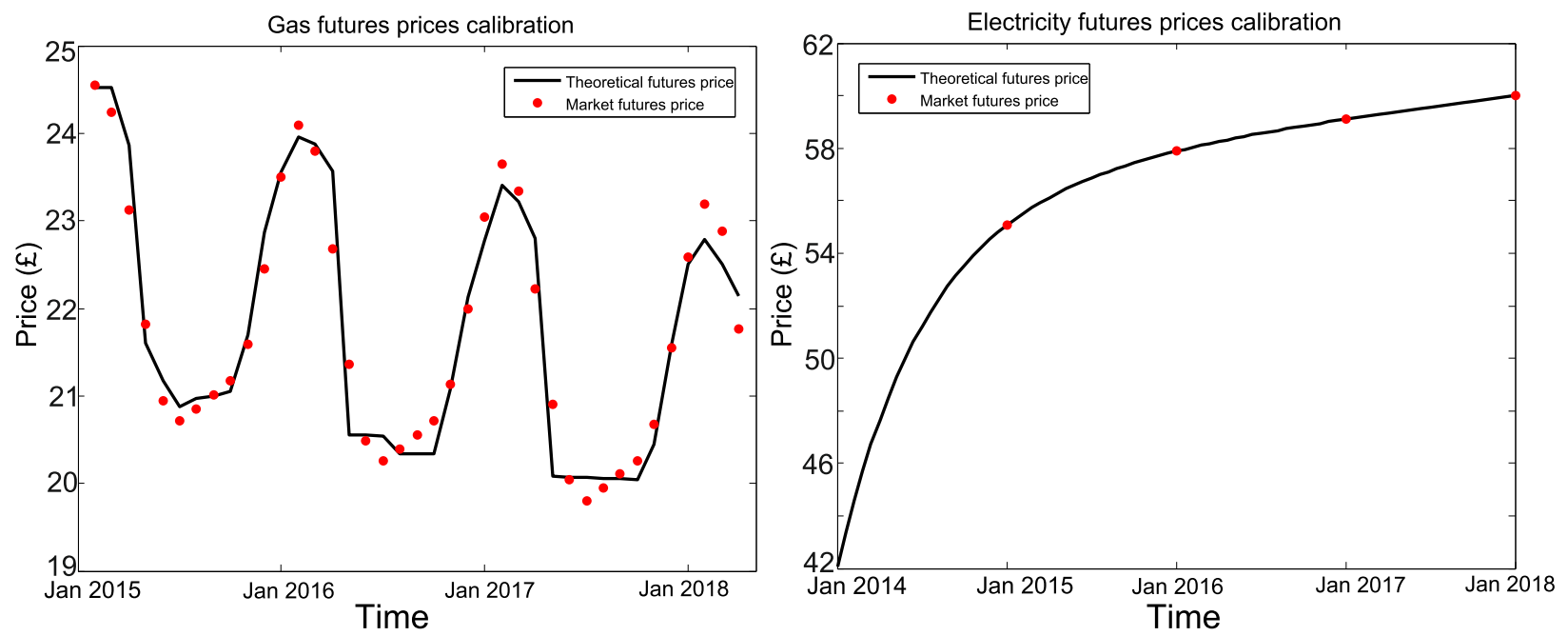

Figure 3: Quoted futures prices and fitted theoretical futures prices for gas (left) and electricity (right). The data series are described in detail in section 4.2.

$\mu_{g}=-0.454$ and seasonal component

$$
\begin{aligned}
f(t)= & 22.83+1.73 \cos (2 \pi t / 8760) \\
& +0.44 \cos (4 \pi t / 8760)+0.21 \sin (4 \pi t / 8760)
\end{aligned}
$$

(note that three years is equal to 8760 hours). As can be seen from Fig. 3, a good fit is achieved between theoretical and observed futures prices.

\section{Results and discussion}

\subsection{Real option value of heat storage}

Table 5 presents the average total operational cost over the three years 2015 to 2017 inclusive for our case study district energy system under rational (that is, optimal) management, together with sensitivity analyses for the heat storage capacity, electricity price drift and gas price drift with central values $15 \mathrm{MWh}$, $\mu_{e}$ and $\mu_{g}$ respectively. While the central price drifts have been calibrated (see section 4.4), the central storage capacity of $15 \mathrm{MWh}$ was chosen for illustration.
The real option value of the operational flexibility provided by the $15 \mathrm{MWh}$ heat store in our case study is given by the decrease in expected operational cost over three years for the $15 \mathrm{MWh}$ store relative to the $0 \mathrm{MWh}$ base case (when the store is not present or is not used), a saving of $£ 104,000$. These savings increase and eventually saturate as the capacity of the heat store increases. Our results confirm that imposing a daily operational cycle is suboptimal in the sense that it reduces this real option value: if the storage level is constrained to return to $12 \mathrm{MWh}$ each day at midnight the real option value is reduced by $£ 7,000$. The real option value of storage is also seen to be robust with respect to variations in the assumptions on long-term energy price trends. As can be seen from table 5 , relative variations of $+/-50 \%$ in the assumptions about the long-term price trends $\mu_{e}$, $\mu_{g}$ (estimated in section 4.4) result in changes which are firstly significantly smaller than the real option value itself (between $£ 24,000$ and $£ 30,000$ over the three year period), and which secondly are almost symmetric. This approximately linear relationship between price trend and operational cost means, for example, that if these different price drifts are treated 


\section{Table 2}

Expected operational cost over three years under the optimal control strategy and sensitivity analysis. The default values for heat storage capacity, electricity price drift and gas price drift are $15 \mathrm{MWh}, \mu_{e}$ and $\mu_{g}$ respectively (see section 4.4). Along each row one of these parameters varies while the other two are fixed at their default values. The final row gives the expected operational cost when the storage level is constrained to return to $12 \mathrm{MWh}$ at midnight each day.

\begin{tabular}{lccccc}
\hline \hline Heat storage capacity & $0 \mathrm{MWh}$ & $7.5 \mathrm{MWh}$ & \multicolumn{2}{c}{$15 \mathrm{MWh}$} & $22.5 \mathrm{MWh}$ \\
Operational cost, MM & 2.238 & 2.153 & \multicolumn{2}{c}{2.134} & 2.126 \\
\hline \hline Gas price drift & & $\mu_{g} / 2$ & $\mu_{g}$ & $3 \mu_{g} / 2$ \\
Operational cost, MM & & 2.164 & 2.134 & 2.103 \\
Electricity price drift & & $\mu_{e} / 2$ & $\mu_{e}$ & $3 \mu_{e} / 2$ & \\
Operational cost, MM & & 2.158 & 2.134 & 2.109 \\
\hline \hline Constrained operational cost, MM & & \multicolumn{3}{c}{2.141} & \\
\hline \hline
\end{tabular}

as scenarios with symmetric probability weights, the average expected cost across all scenarios remains close to the central real option value indicated above of $£ 104,000$ over three years. The real option value of the heat store is therefore robust with respect to our price drift assumptions.

\subsection{Interpretation of control maps}

Examples of the obtained control maps are illustrated in Fig. 4. The map at any given point in time takes the form of a scatter plot with $\mathrm{N}$ points, where $\mathrm{N}$ is the number of price paths sampled (see section 3.2 ). In this way the resolution of our control maps is guided by the statistical price models themselves, the control strategy becoming more detailed in regions where the prices are found more often. Although these control maps vary appreciably across different hourly periods within the same day, they show strong similarity across days at the same hourly period (and within the same season). This feature can be anticipated from the stochastic price models used for electricity and gas, which are statistically stationary across days at the same hour, and the demand profiles which follow diurnal cycles. Indeed, the maps in Fig. 4 have been obtained by superimposing the control maps at the same hour (10am) from 20 consecutive days in the planning period, for the two different seasons (winter on the left and summer on the right).
(Taking the opposite cross-section by superimposing the 24 control maps within a single day does not in general produce a clear picture, confirming the nonstationarity within single days). In this way we have obtained significantly clearer and more detailed control maps than those reported in [12]: since in each map the markers are located at $N$ random points generated by our Monte Carlo simulation of price paths, these locations are in general different across days and their superimposition gives a more dense map; however, statistical stationarity means that maps for the same hour and within the same season, but on adjacent days, may be assumed to represent the same strategy and so may be meaningfully superimposed to obtain higher resolution.

The control maps shown in Fig. 4 are typical for this case study in that they are clear and highly interpretable. It may be seen from Fig. 4 that the CHP unit's control strategy simply acts to exploit the diurnal and stochastic variations in the spark spread. The winter boiler strategy (Fig. 4, top left) serves to exploit gas prices by generating heat at maximum rate when the gas price is below average, while during periods of above-average gas price it acts in sympathy with the CHP unit, decreasing the boiler's power as the spark spread increases and the CHP unit accordingly cogenerates increasing amounts of heat. 

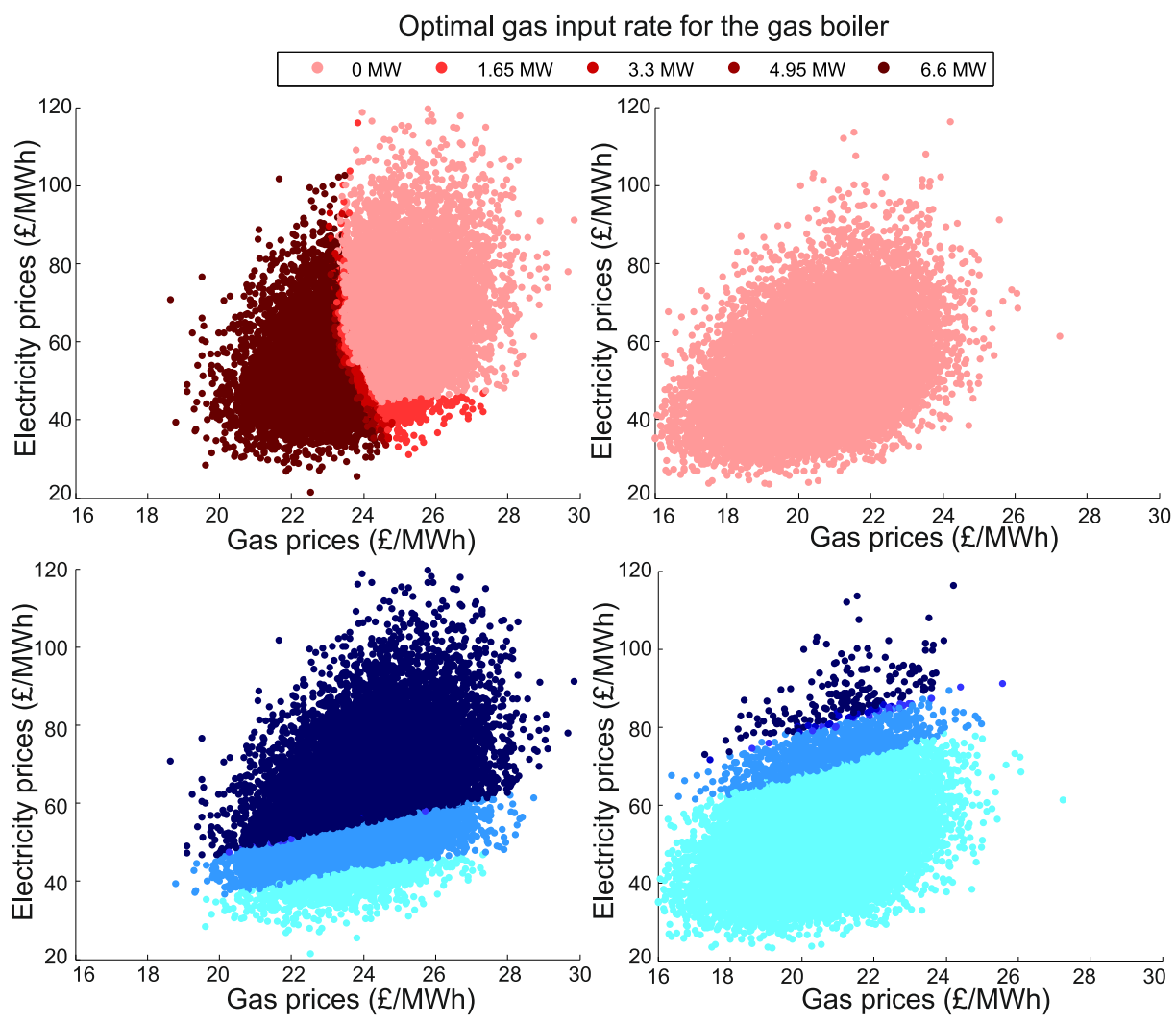

Optimal gas input rate for the CHP unit

$0 \mathrm{MW} \bullet 1.875 \mathrm{MW} \bullet 3.75 \mathrm{MW} \bullet 5.625 \mathrm{MW} \quad \bullet \quad 7.5 \mathrm{MW}$

Figure 4: Optimal operating state for the gas boiler (top) and CHP unit (bottom) in January 2016 at 10 am (left) and in July 2016 at 10 am (right), when the level of stored heat is 4.5 MWh.

\subsection{Inference from quoted futures prices}

The red markers plotted in Fig. 3 illustrate that a high degree of structure was present in the quoted futures prices for electricity and gas, indicating a potential to make clear inferences on the market's view of anticipated price trends. Further, the fitted curves in Fig. 3 show that it is possible to successfully calibrate standard commodity price models from mathematical finance to these prices. For gas futures the price variation is dominated by the seasonal price cycle and an approximately linear decreasing trend. The electricity futures studied exhibit an approximately constant summer/winter price differential, and the logarithm of the futures prices exhibits an approximately linear increasing trend. At present these expected trends are increasing for electricity prices and decreasing for gas prices (section 4.4). As mentioned above, the sensitivity analysis given in table 5 shows that 
even changing these growth assumptions on gas and electricity prices by $+/-50 \%$ does not significantly change the value of the heat storage unit (this value is the difference in expected total operational costs with and without heat storage). Indeed, if electricity prices grow faster than expected this increases the value of storage as the CHP unit makes increasing returns from exploitation of the spark spread; similarly, if gas prices fall faster due for example to an expansion in supply from UK shale gas then the value of storage again increases. Again this highlights how thermal energy storage would be a robust solution to enhance energy system flexibility.

\subsection{Regression procedure and compu- tational speed}

The crucial role played by dynamic programming in our method is clear when we consider that, for each given hour, the number of possible control maps such as Fig. 4 grows as $n_{1}^{n_{2} n_{3}}$, where $n_{1}$ is the total number of operational modes, $n_{2}$ is the total number of possible electricity-gas price pairs, and $n_{3}$ is the number of time periods in the optimisation. Even taking $n_{3}=24$, a 'brute force' exhaustive search performed by simulating the system's performance under each possible dynamic feedback control strategy is infeasible and it is for this reason that we employ dynamic programming. A heuristic search guided by the experience of typical optimal control strategies is possible in principle, but as we show in this section the speed of our dynamic programming procedure makes it suitable as an online, real-time optimisation tool and heuristic approximation is not needed.

The regression step addresses the fact that sampling 1000 independent price sample paths only provides an approximation to the probability measure governing these price paths. We note that the regression step can be excluded and a more classical dynamic programming procedure performed instead, with a corresponding decrease in computational time and complexity; this is however problematic, since re-sampling another set of 1000 paths in exactly the same way would lead to different results. The regression step aims to separate the systematic variation in average cost from the noise introduced by Monte
Carlo simulation, and thus to provide results which are more consistently reproducible.

The consistency of these results may be checked by direct simulation of the district energy system under the obtained optimal strategy. In order to estimate the numerical error introduced by simulation, the same set of simulated electricity and gas price paths generated during optimisation are used in a forward simulation of the operational cost. Since the dynamic programming procedure is recursive there is error propagation over time, and in our case study with a time horizon of three years the difference in value functions is approximately $10 \%$; in contrast, over two years, one year and one-week optimisations the differences in value functions are approximately $6 \%, 3 \%$ and $0.07 \%$ respectively (data not shown).

Using 1000 Monte Carlo sample paths we are able to return both the total expected operational cost and separate operational control maps for the CHP and boiler units for each of the 26280 hourly periods, in the presence of stochastic electricity and gas prices, within 10.5 hours on a 3.7 GhZ Intel Xeon server with $12 \mathrm{MB}$ RAM. This running time is linear with respect to the length of the optimisation period so that, for example, a one week optimisation would take four minutes with the same machine and it is clear that short-term operational control strategies can be recalculated in real time. Running times can be further reduced by reducing the number of sample paths, at the expense of a loss of accuracy in general.

\subsection{Considerations on policy instru- ments}

It has to be highlighted that in the analysis performed here we have not taken into account policy issues and relevant instruments that in reality could influence the business case of low carbon technologies and district energy systems as in our case study. Such instruments may include incentives for high efficiency electricity generation (as in many countries for CHP plants), carbon taxes, feed in tariffs, and so forth. For instance, depending on the country-specific policy settings, market prices (e.g., due to carbon taxes or tradable emission permits) or the district energy system's cash flows (e.g., due to subventions for high 
efficiency CHP) might change. The reason to exclude policy instruments is that the focus of this work is more on modelling and assessing the role of thermal storage as an operational flexibility enabler to exploit the different forms of arbitrage highlighted above, rather than on developing a full business case for district energy systems per se. However, given the set of policy instruments present in a given country, the model developed could be readily adapted to include those too, for instance by considering market prices that already take account of internalisation of externalities for example through carbon taxes, or by including the relevant cash flow changes in the case of specific incentives for the system under study. On the other hand, policy instruments may represent an additional source of uncertainty (particularly in the long term) relevant to the planning aspects, which could be incorporated in the framework developed for instance by running different policy scenarios and overlaying either probabilistic or robust scenario analysis. This is in fact the object of work in progress.

\section{Conclusion}

In this paper, we have proposed a model for optimal and dynamic control and long term valuation of CHP-thermal storage in the presence of uncertain market prices. The proposed model has been formulated as a stochastic linear constrained control problem and numerically solved through Least Squares Monte Carlo regression analysis. Analysis of investment and operational timescales has been dealt with in an integrated manner, so that the model provides at the same time optimal control strategies for real time demand response to market signals and real options valuation of thermal storage as major enabler of flexibility. We have shown that significant performance increases are possible by exploiting statistical stationarity: by allowing the statistical price models themselves to guide the resolution of the control strategy we obtain higher-quality information where it is needed most, at the most likely system states, at the expense of a linear increase in computational time. Amongst others, novel graphical outputs from the model are represented by clear and interpretable feedback control strategy maps for each hour of the day that can be deployed for real time decisions that exploit both the gas-electricity spark spread and real time market prices. Numerical applications to a realistic UK district energy system with CHP, back-up boiler and thermal storage have been shown to exemplify the proposed approach. In order to allow realistic assessment and valuation, gas and electricity prices have been modelled as stochastic processes and calibrated on available futures contract prices. The performance of the approach has been tested in a three-year example case, examining sensitivity of the projected operational cost under optimal control to both energy price trends and the installed storage capacity. The findings indicate that the operating cost reductions offered by heat storage are robust, even in scenarios where gas and electricity price trends are unfavourable relative to the current implied UK market view. The approximation error due to the regression part of the procedure has also been estimated via a forward Monte Carlo simulation, indicating that the obtained average operational costs are near-identical over operational timescales and remain close even over very long timescales. From this work it is possible to clearly highlight how the real time control maps that are drawn allow real-time decision making while uncertainty (in this case in energy prices, but it could be generalised to other variables) unfolds over time, thus being applicable in practical controllers within a Smart Grid operational framework with real-time information on energy prices, consumption, and so on. In addition, our findings clearly indicate that thermal storage can be a key component to enable flexibility in district energy systems and thus improve the business case of such high efficiency solutions.

\section{Acknowledgements}

YK was supported by EPSRC grant EP/I01912X/1, JM was supported by EPSRC grant EP/K00557X/1, $\mathrm{JM}$ and PM were supported by EPSRC grant $\mathrm{EP} / \mathrm{I} 031650 / 1$. 


\section{References}

[1] Mancarella P. MES (multi-energy systems): an overview of concepts and evaluation models. Invited Paper, Energy 2014;65(1):117.

[2] Mancarella P, Chicco G. Distributed multigeneration systems. Energy models and analyses, Nova Science Publishers, Hauppauge, NY, 2009.

[3] Chicco G, Mancarella P. Distributed multigeneration: A comprehensive view. Renewable and Sustainable Energy Reviews 2009;13(3):535551

[4] Mancarella P, Chicco G. Real-time demand response from energy shifting in Distributed MultiGeneration. IEEE Transactions on Smart Grid 2013;4(4):1928-1938.

[5] Capuder T, Mancarella P. Techno-economic and Environmental Modelling and Optimization of Flexible Distributed Multi-Generation Options. Energy, Accepted for publication, 2014.

[6] Lund H, Andersen AN. Optimal designs of small CHP plants in a market with fluctuating electricity prices. Energy Convers. Manag. 2005;46:893904.

[7] Rolfsman B. Combined heat-and-power plants and district heating in a deregulated electricity market. Appl. Energy 2004;78:3752.

[8] Fragaki A, Andersen AN. Conditions for aggregation of CHP plants in the UK electricity market and exploration of plant size. Appl. Energy. 2011;88:39303940.

[9] Fragaki A, Andersen AN, Toke D. Exploration of economical sizing of gas engine and thermal store for combined heat and power plants in the UK. Energy 2008;33:16591670.

[10] Carpaneto E, Chicco G, Mancarella P, Russo A. Cogeneration planning under uncertainty. Part I: Multiple time frame approach. Applied Energy 2011;88(4):1059-1067.
[11] Carpaneto E, Chicco G, Mancarella P, Russo A. Cogeneration planning under uncertainty. Part II: Decision theory-based assessment of planning alternatives. Applied Energy 2011;88(4)

[12] Kitapbayev Y, Moriarty J, Mancarella P, Blochle M. A real option assessment of operational flexibility in district energy systems. European Energy Markets (EEM) Conference 2013.

[13] Carmona R, Ludkowski M. Valuation of Energy Storage: An Optimal Switching Approach. Quantitative Finance 2010;10(4): 359-374.

[14] Dixit RK, Pindyck RS. Investment under uncertainty, Princeton university press 2008.

[15] Australian Energy Regulator, Regulatory investment test for transmission application guidelines, 2010.

[16] Amram M, Kulatilaka NH. Real Options: Managing Strategic Investment in an Uncertain World, OUP Catalogue, 2012.

[17] Trigeorgis L. Real options: Managerial flexibility and strategy in resource allocation. MIT press, 1996.

[18] Kienzle F, Ahcin P, Andersson G. Valuing investments in multi-energy conversion, storage, and demand-side management systems under uncertainty, IEEE Trans. Sust. Energy 2011;2(2):194202.

[19] Fleming W, Rishel R. Deterministic and stochastic optimal control. Springer, 1975.

[20] Longstaff FA, Schwartz ES. Valuing American options by simulation: A simple leastsquares approach. Review of Financial studies 2001;14(1):113-147.

[21] University of Strathclyde, "Demand Profile Generator" [Online]. Available at: www.esru.strath.ac.uk/EandE/Web_sites/ 06-07/Carbon_neutral/toolsfolder/ DemandProfileGenerators.htm. 
[22] Benth FE, Benth JvS, Koekebakker S. Stochastic modelling of electricity and related markets. World Scientific Publishing Company Incorporated, vol. 11, 2008. 